\title{
Research Article \\ Fixed Point of Strong Duality Pseudocontractive Mappings and Applications
}

\author{
Baowei Liu \\ Department of Mathematics, Cangzhou Normal University, Cangzhou 061001, China \\ Correspondence should be addressed to Baowei Liu, lbw8880148@sina.com
}

Received 16 July 2012; Accepted 22 July 2012

Academic Editor: Yongfu Su

Copyright (C) 2012 Baowei Liu. This is an open access article distributed under the Creative Commons Attribution License, which permits unrestricted use, distribution, and reproduction in any medium, provided the original work is properly cited.

Let $E$ be a smooth Banach space with the dual $E^{*}$, an operator $T: E \rightarrow E^{*}$ is said to be $\alpha$-strong duality pseudocontractive if $\langle x-y, T x-T y\rangle \leq\langle x-y, J x-J y\rangle-\alpha\|J x-J y-(T x-T y)\|^{2}$, for all $x, y \in E$, where $\alpha$ is a nonnegative constant. An element $x \in E$ is called a duality fixed point of $T$ if $T x=J x$. The purpose of this paper is to introduce the definition of $\alpha$-strong duality pseudocontractive mappings and to study its fixed point problem and applications for operator equation and variational inequality problems.

\section{Introduction and Preliminaries}

Let $E$ be a real Banach space with the dual $E^{*}$ : let $T$ be an operator from $E$ into $E^{*}$. We consider the first operator equation problem of finding an element $x^{*} \in E$ such that

$$
\left\langle T x^{*}, x^{*}\right\rangle=\left\|T x^{*}\right\|^{2}=\left\|x^{*}\right\|^{2} .
$$

We also consider the second variational inequality problem of finding an element $x^{*} \in E$ such that

$$
\left\langle T x^{*}, x^{*}-x\right\rangle \geq 0, \quad \forall\|x\| \leq\left\|x^{*}\right\| .
$$

Let $E$ be a real Banach space with the dual $E^{*}$. Let $p$ be a given real number with $p>1$. The generalized duality mapping $J_{p}$ from $E$ into $2^{E^{*}}$ is defined by

$$
J_{p}(x)=\left\{f \in E^{*}:\langle x, f\rangle=\|f\|^{p},\|f\|=\|x\|^{p-1}\right\}, \quad \forall x \in E,
$$


where $\langle\cdot, \cdot\rangle$ denotes the generalized duality pairing. In particular, $J=J_{2}$ is called the normalized duality mapping and $J_{p}(x)=\|x\|^{p-2} J(x)$ for all $x \neq 0$. If $E$ is a Hilbert space, then $J=I$, where $I$ is the identity mapping. The duality mapping $J$ has the following properties:

(i) if $E$ is smooth, then $J$ is single valued;

(ii) if $E$ is strictly convex, then $J$ is one to one;

(iii) if $E$ is reflexive, then $J$ is a mapping of $E$ onto ${ }^{*} E$;

(iv) if $E$ is uniformly smooth, then $J$ is uniformly norm-to-norm continuous on each bounded subset of $E$;

(v) if $E^{*}$ is uniformly convex, then $J$ is uniformly continuous on each bounded subsets of $E$ and $J$ is single valued and also one to one.

For more details, see $[1,2]$.

Let $E$ be a smooth Banach space with the dual $E^{*}$. Let $T: E \rightarrow E^{*}$ be an operator; an element $x^{*} \in E$ is called a duality fixed point of $T$, if $T x^{*}=J x^{*}$. such that

We also consider the third variational inequality problem of finding an element $x^{*} \in E$

$$
\left\langle T x^{*}, x-x^{*}\right\rangle \geq 0, \quad \forall x \in C,
$$

where $C$ is a closed convex subset of $E$. The set of solutions of the variational inequality problem (1.4) is denoted by $V I(C, T)$. E such that

We also consider the fourth variational inequality problem of finding an element $x^{*} \in$

$$
\left\langle J x^{*}-T x^{*}, x-x^{*}\right\rangle \geq 0, \quad \forall x \in C,
$$

where $C$ is a closed convex subset of $E$. The set of solutions of the variational inequality problem (1.5) is denoted by $V I(C, J, T)$.

Conclusion 1. If $x^{*}$ is a duality fixed point of $T$, then $x^{*}$ must be a solution of problem (1.1).

Proof. If $x^{*}$ is a normalized fixed point of $T$, then $T x^{*}=J x^{*}$, so that

$$
\left\langle T x^{*}, x^{*}\right\rangle=\left\langle J x^{*}, x^{*}\right\rangle=\left\|J x^{*}\right\|^{2}=\left\|T x^{*}\right\|^{2}=\left\|x^{*}\right\|^{2} .
$$

This completes the proof.

Conclusion 2. If $x^{*}$ is a duality fixed point of $T$, then $x^{*}$ must be a solution of variational inequality problem (1.2).

Proof. Suppose $x^{*}$ is a duality fixed point of $T$; then

$$
\left\langle T x^{*}, x^{*}\right\rangle=\left\|T x^{*}\right\|^{2}=\left\|x^{*}\right\|^{2} .
$$


Obverse that

$$
\begin{aligned}
\left\langle T x^{*}, x^{*}-x\right\rangle & =\left\langle T x^{*}, x^{*}\right\rangle-\left\langle T x^{*}, x\right\rangle \\
& \geq\left\|T x^{*}\right\|^{2}-\left\|T x^{*}\right\|\|x\| \\
& =\left\|T x^{*}\right\|\left(\left\|T x^{*}\right\|-\|x\|\right) \\
& =\left\|T x^{*}\right\|\left(\left\|x^{*}\right\|-\|x\|\right) \geq 0,
\end{aligned}
$$

for all $\|x\| \leq\left\|x^{*}\right\|$. This completes the proof.

Let $U=\{x \in E:\|x\|=1\}$. A Banach space $E$ is said to be strictly convex if for any $x, y \in U, x \neq y$ implies $\|(x+y) / 2\|<1$. It is also said to be uniformly convex if for each $\varepsilon \in(0,2]$, there exists $\delta>0$ such that for any $x, y \in U,\|x-y\| \geq \varepsilon$ implies $\|(x+y) / 2\|<1-\delta$. It is known that a uniformly convex Banach space is reflexive and strictly convex. And we define a function $\delta:[0,2] \rightarrow[0,1]$ called the modulus of convexity of $E$ as follows:

$$
\delta(\varepsilon)=\left\{1-\left\|\frac{x+y}{2}\right\|:\|x\|=\|y\|=1,\|x-y\| \geq \varepsilon\right\} .
$$

It is known that $E$ is uniformly convex if and only if $\delta(\varepsilon)>0$ for all $\varepsilon \in(0,2]$. Let $p$ be a fixed real number with $p \geq 2$. Then $E$ is said to be $p$-uniformly convex if there exists a constant $c>0$ such that $\delta(\varepsilon) \geq c \varepsilon^{p}$ for all $\varepsilon \in[0,2]$. For example, see [3,4] for more details. The constant $1 / c$ is said to be uniformly convexity constant of $E$.

A Banach space $\mathrm{E}$ is said to be smooth if the limit

$$
\lim _{t \rightarrow 0} \frac{\|x+t y\|-\|x\|}{t}
$$

exists for all $x, y \in U$. It is also said to be uniformly smooth if the above limit is attained uniformly for $x, y \in U$. One should note that no Banach space is $p$-uniformly convex for $1<p<2$; see [5] for more details. It is well known that the Hilbert and the Lebesgue $L^{q}(1<$ $q \leq 2)$ spaces are 2-uniformly convex and uniformly smooth. Let $X$ be a Banach space, and let $L^{q}(X)=\{\Omega, \Sigma, \mu ; X\}, 1<q \leq \infty$ be the Lebesgue-Bochner space on an arbitrary measure space $(\Omega, \Sigma, \mu)$. Let $2 \leq p<\infty$, and let $1<q \leq p$. Then $L^{q}(X)$ is $p$-uniformly convex if and only if $X$ is $p$-uniformly convex; see [4].

In this paper, we first propose the definition of generalized $\alpha$-strongly pseudocontractive mappings from a smooth Banach $E$ into its dual $E^{*}$ as follows. We also discuss the problem of fixed point for generalized $\alpha$-strongly pseudocontractive mappings and its applications.

Let $E$ be a smooth Banach space and $E^{*}$ denote the dual of $E$. An operator $A: E \rightarrow E^{*}$ is said to be

(1) $\alpha$-inverse-strongly monotone if there exists nonnegative real number $\alpha$ such that

$$
\langle x-y, A x-A y\rangle \geq \alpha\|A x-A y\|^{2}, \quad \forall x, y \in E .
$$


(2) $\alpha$-strong duality pseudocontractive mapping, if there exists a nonnegative real number $\alpha$ such that

$$
\langle x-y, A x-A y\rangle \leq\langle x-y, J x-J y\rangle-\alpha\|J x-J y-(A x-A y)\|^{2}
$$

for all $x, y \in E$.

It is easy to show that $A$ is $\alpha$-strong duality pseudocontractive if and only if $(J-A)$ is $\alpha$-inverse-strongly monotone.

Let $E$ be a smooth Banach space and $E^{*}$ denote the dual of $E$. Let $A: E \rightarrow E^{*}$ be an operator. The set of zero points of $A$ is defined by $A^{-1} 0=\{x \in E: A x=0\}$. The set of duality fixed points of $A$ is defined by $F(A)=\{x \in E: A x=J x\}$. It is also easy to show that, an element $u \in E$ is a zero point of an $\alpha$-inverse-strongly monotone operator $A$ if and only if $u$ is a duality fixed point of the $\alpha$-strong duality pseudocontractive mapping $(J-A)$.

\section{Main Results and Applications}

Recently, Zegeye and Shahzad [6] proved the following result.

Theorem 2.1 (see, [6]). Let E be a uniformly smooth and 2-uniformly convex real Banach space with the dual $E^{*}:$ let $A: E \rightarrow E^{*}$ be a $\gamma$-inverse-strongly monotone mapping and $T: E \rightarrow E$ a relatively weak nonexpansive mapping with $A^{-1} 0 \cap F(T) \neq \emptyset$. Assume that $0<\alpha \leq \lambda_{n} \leq \gamma c^{2} / 2$, where $1 / c$ is the uniformly convexity constant. Define a sequence $\left\{x_{n}\right\}$ in $E$ by the following algorithm:

$$
\begin{gathered}
x_{0} \in E \text { chosen arbitrarily, } \\
z_{n}=J^{-1}\left(J x_{n}-\lambda_{n} A x_{n}\right), \\
y_{n}=T z_{n}, \\
C_{n}=\left\{z \in C_{n-1} \bigcap Q_{n-1}: \phi\left(z, y_{n}\right) \leq \phi\left(z, z_{n}\right) \leq \phi\left(z, x_{n}\right)\right\}, \\
C_{0}=\left\{z \in E: \phi\left(z, y_{0}\right) \leq \phi\left(z, z_{0}\right) \leq \phi\left(z, x_{0}\right)\right\}, \\
Q_{n}=\left\{z \in C_{n-1} \bigcap Q_{n-1}:\left\langle x_{n}-z, J x_{0}-J x_{n}\right\rangle \geq 0\right\}, \\
Q_{0}=E, \\
x_{n+1}=\prod_{C_{n} \cap Q_{n}}\left(x_{0}\right),
\end{gathered}
$$

where $J$ is the duality mapping on $E$. Then $\left\{x_{n}\right\}$ converges strongly to $\Pi_{A^{-1} 0 \cap F(T)} x_{0}$, where $\Pi_{A^{-1} 0 \cap F(T)}$ is the generalized projection from $E$ onto $A^{-1} 0 \cap F(T)$.

IF taking $T=I$, then Theorem 2.1 reduces to the following result.

Theorem 2.2. Let $E$ be a uniformly smooth and 2-uniformly convex real Banach space with the

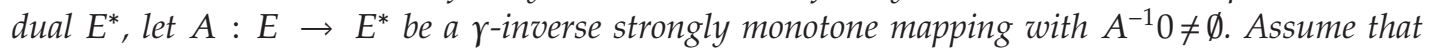


$0<\alpha \leq \lambda_{n} \leq \gamma c^{2} / 2$, where $1 / c$ is the uniformly convexity constant. Define a sequence $\left\{x_{n}\right\}$ in $E$ by the following algorithm:

$$
\begin{gathered}
x_{0} \in E \text { chosen arbitrarily, } \\
y_{n}=J^{-1}\left(J x_{n}-\lambda_{n} A x_{n}\right), \\
C_{n}=\left\{z \in C_{n-1} \bigcap Q_{n-1}: \phi\left(z, y_{n}\right) \leq \phi\left(z, x_{n}\right)\right\}, \\
C_{0}=\left\{z \in E: \phi\left(z, y_{0}\right) \leq \phi\left(z, x_{0}\right)\right\}, \\
Q_{n}=\left\{z \in C_{n-1} \bigcap Q_{n-1}:\left\langle x_{n}-z, J x_{0}-J x_{n}\right\rangle \geq 0\right\}, \\
Q_{0}=E, \\
x_{n+1}=\prod_{C_{n} \cap Q_{n}}\left(x_{0}\right),
\end{gathered}
$$

where $J$ is the duality mapping on $E$. Then $\left\{x_{n}\right\}$ converges strongly to $\Pi_{A^{-1} 0} x_{0}$, where $\Pi_{A^{-1} 0}$ is the generalized projection from $E$ onto $A^{-1} 0$.

Theorem 2.3. Let $E$ be a uniformly smooth and 2-uniformly convex real Banach space; let $A: E \rightarrow$ $E^{*}$ be an $\alpha$-strong duality pseudocontractive mapping with nonempty set of duality fixed points $F(A)$. Let $T: E \rightarrow E$ be a relatively weak nonexpansive mapping and $F(A) \cap F(T)=\emptyset$. Assume $0<a \leq$ $\lambda_{n} \leq \alpha c^{2} / 2 L$. Define a sequence $\left\{x_{n}\right\}$ in $E$ by the following algorithm:

$$
\begin{gathered}
x_{0} \in E \text { chosen arbitrarily, } \\
z_{n}=J^{-1}\left(\left(1-\lambda_{n}\right) J x_{n}+\lambda_{n} A x_{n}\right), \\
y_{n}=T z_{n} \\
C_{n}=\left\{z \in C_{n-1} \bigcap Q_{n-1}: \phi\left(z, y_{n}\right) \leq \phi\left(z, z_{n}\right) \leq \phi\left(z, x_{n}\right)\right\}, \\
C_{0}=\left\{z \in E: \phi\left(z, y_{0}\right) \leq \phi\left(z, z_{0}\right) \leq \phi\left(z, x_{0}\right)\right\}, \\
Q_{n}=\left\{z \in C_{n-1} \bigcap Q_{n-1}:\left\langle x_{n}-z, J x_{0}-J x_{n}\right\rangle \geq 0\right\}, \\
Q_{0}=E, \\
x_{n+1}=\prod_{C_{n} \cap Q_{n}}\left(x_{0}\right),
\end{gathered}
$$

where $J$ is the duality mapping on $E$. Then $\left\{x_{n}\right\}$ converges strongly to a common element $x^{*} \in$ $F(A) \cap F(T)$. This element is also a common solution of operator equation (1.1) and variational inequality (1.2).

Proof. Let $B=J-A$, then $B: E \rightarrow E^{*}$ is $\alpha / L$-inverse-strongly monotone and $\alpha$-strongly monotone, so that $B^{-1} 0=F(A)$ has only one element. On the other hand, we have

$$
z_{n}=J^{-1}\left(\left(1-\lambda_{n}\right) J x_{n}+\lambda_{n} A x_{n}\right)=J^{-1}\left(J x_{n}-\lambda_{n} B x_{n}\right) .
$$

By using Theorem 2.1 and Conclusions 1 and 2, we obtain the conclusion of Theorem 2.3. Taking $T=I$ in Theorem 2.3, we get the following result. 
Theorem 2.4. Let $E$ be a uniformly smooth and 2-uniformly convex real Banach space; let $A: E \rightarrow$ $E^{*}$ be a L-Lipschitz and $\alpha$-strongly duality pseudocontractive mapping with nonempty set of duality fixed points $F(A)$. Assume $0<a \leq \lambda_{n} \leq \alpha c^{2} / 2 L$. Define a sequence $\left\{x_{n}\right\}$ in E by the following algorithm:

$$
\begin{gathered}
x_{0} \in E \text { chosen arbitrarily, } \\
y_{n}=J^{-1}\left(\left(1-\lambda_{n}\right) J x_{n}+\lambda_{n} A x_{n}\right), \\
C_{n}=\left\{z \in C_{n-1} \bigcap Q_{n-1}: \phi\left(z, y_{n}\right) \leq \phi\left(z, x_{n}\right)\right\}, \\
C_{0}=\left\{z \in E: \phi\left(z, y_{0}\right) \leq \phi\left(z, x_{0}\right)\right\}, \\
Q_{n}=\left\{z \in C_{n-1} \bigcap Q_{n-1}:\left\langle x_{n}-z, J x_{0}-J x_{n}\right\rangle \geq 0\right\}, \\
Q_{0}=E, \\
x_{n+1}=\Pi_{C_{n} \cap Q_{n}}\left(x_{0}\right),
\end{gathered}
$$

where $J$ is the duality mapping on $E$. Then $\left\{x_{n}\right\}$ converges strongly to a duality fixed point $x^{*} \in F(A)$. This element $x^{*}$ is also a common solution of operator equation (1.1) and variational inequality (1.2).

liduka and Takahashi [7] introduce an iterative scheme for finding a solution of the variational inequality problem for an operator $A$ that satisfies the following conditions (i)(iii) in a 2-uniformly convex and uniformly smooth Banach space $E$ :

(i) $A$ is $\alpha$-inverse-strongly monotone;

(ii) $\operatorname{VI}(C, A) \neq \emptyset$;

(iii) $\|A y\| \leq\|A y-A u\|$ for all $y \in E$ and $u \in V I(C, A)$.

They proved the following convergence theorem.

Theorem 2.5 (see, [7]). Let E be a 2-uniformly convex and uniformly smooth Banach space, whose duality mapping $J$ is weakly sequentially continuous, and $C$ a nonempty, closed convex subset of E. Assume that $A$ is an operator of $C$ into $E^{*}$, that satisfies the conditions (i)-(iii). Suppose that $x_{1}=x \in C$ and $\left\{x_{n}\right\}$ is given by

$$
x_{n+1}=\Pi_{C} J^{-1}\left(J x_{n}-\lambda_{n} A x_{n}\right)
$$

for every $n=1,2, \ldots$, where $\left\{\lambda_{n}\right\}$ is a sequence of positive numbers. If $\left\{\lambda_{n}\right\}$ is chosen so that $\lambda_{n} \in[a, b]$ for some $a, b$ with $0<a<b<c^{2} \alpha / 2$, then the sequence $\left\{x_{n}\right\}$ converges weakly to some element $z \in$ $V I(C, A)$, where $1 / c$ is the 2-uniformly convexity constant of $E$. Further $z=\lim _{n \rightarrow \infty} \Pi_{V I(C, A)} x_{n}$.

In this paper, we introduce an iterative scheme for finding a solution of the variational inequality problem for an operator $T$ that satisfies the following conditions (iv)-(vi) in a 2uniformly convex and uniformly smooth Banach space $E$ :

(iv) $T$ is $\alpha$-strong duality pseudocontractive,

(v) $V I(C, J, T) \neq \emptyset$,

(vi) $\|J y-T y\| \leq\|(J-T) y-(J-T) u\|$ for all $y \in E$ and $u \in V I(C, J, T)$.

By using Theorem 2.5, we prove the following convergence theorem. 
Theorem 2.6. Let E be a 2-uniformly convex and uniformly smooth Banach space, whose duality mapping $J$ is weakly sequentially continuous, and $C$ a nonempty, closed convex subset of $E$. Assume that $T$ is an operator of $C$ into $E^{*}$. that satisfies the conditions (iv)-(vi). Suppose that $x_{1}=x \in C$ and $\left\{x_{n}\right\}$ is given by

$$
x_{n+1}=\Pi_{C} J^{-1}\left(\left(1-\lambda_{n}\right) J x_{n}+\lambda_{n} T x_{n}\right),
$$

for every $n=1,2, \ldots$, where $\left\{\lambda_{n}\right\}$ is a sequence of positive numbers. If $\left\{\lambda_{n}\right\}$ is chosen so that $\lambda_{n} \in[a, b]$ for some $a, b$ with $0<a<b<c^{2} \alpha / 2$, then the sequence $\left\{x_{n}\right\}$ converges weakly to some element $z \in$ $V I(C, J, T)$, where $1 / c$ is the 2 -uniformly convexity constant of $E$. Further $z=\lim _{n \rightarrow \infty} \Pi_{V I(C, J, T)} x_{n}$.

Proof. Let $A=J-T$, then $B: E \rightarrow E^{*}$ is $\alpha$-inverse-strongly monotone, so that $B^{-1} 0=F(A)$. On the other hand, we have

$$
x_{n+1}=\Pi_{C} J^{-1}\left(\left(1-\lambda_{n}\right) J x_{n}+\lambda_{n} T x_{n}\right)=J^{-1}\left(J x_{n}-\lambda_{n} A x_{n}\right) .
$$

By using Theorem 2.5, we obtain the conclusion of Theorem 2.6.

In fact, from condition (vi), we have $F(T)=V I(C, J, T)$, so that under the conditions of Theorem 2.6, the $\left\{x_{n}\right\}$ converges strongly to a duality fixed point $z \in F(T)$. This element $z$ is also a common solution of operator equation (1.1) and variational inequality (1.2). where $\left\{x_{n}\right\}$ is defined by Algorithm (2.7).

\section{References}

[1] W. Takahashi, Nonlinear Functional Analysis, Yokohama Publishers, Yokohama, Japan, 2000.

[2] W. Takahashi, Totsu Kaiseki to Fudoten Kinji, Yokohama Publishers, Yokohama, Japan, 2000.

[3] D. Butnariu, S. Reich, and A. J. Zaslavski, "Asymptotic behavior of relatively nonexpansive operators in Banach spaces," Journal of Applied Analysis, vol. 7, no. 2, pp. 151-174, 2001.

[4] D. Butnariu, S. Reich, and A. J. Zaslavski, "Weak convergence of orbits of nonlinear operators in reflexive Banach spaces," Numerical Functional Analysis and Optimization, vol. 24, no. 5-6, pp. 489-508, 2003.

[5] Y. Takahashi, K. Hashimoto, and M. Kato, "On sharp uniform convexity, smoothness, and strong type, cotype inequalities," Journal of Nonlinear and Convex Analysis, vol. 3, no. 2, pp. 267-281, 2002.

[6] H. Zegeye and N. Shahzad, "Strong convergence theorems for monotone mappings and relatively weak nonexpansive mappings," Nonlinear Analysis, vol. 70, no. 7, pp. 2707-2716, 2009.

[7] H. Iiduka and W. Takahashi, "Weak convergence of a projection algorithm for variational inequalities in a Banach space," Journal of Mathematical Analysis and Applications, vol. 339, no. 1, pp. 668-679, 2008. 


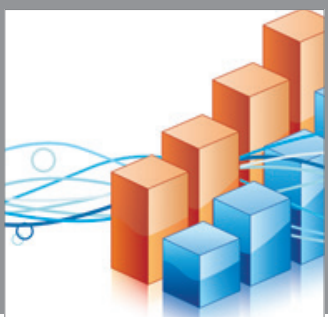

Advances in

Operations Research

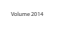

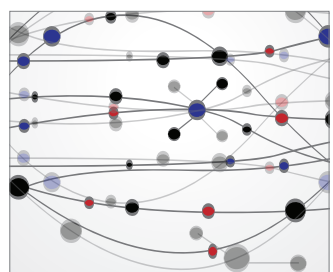

\section{The Scientific} World Journal
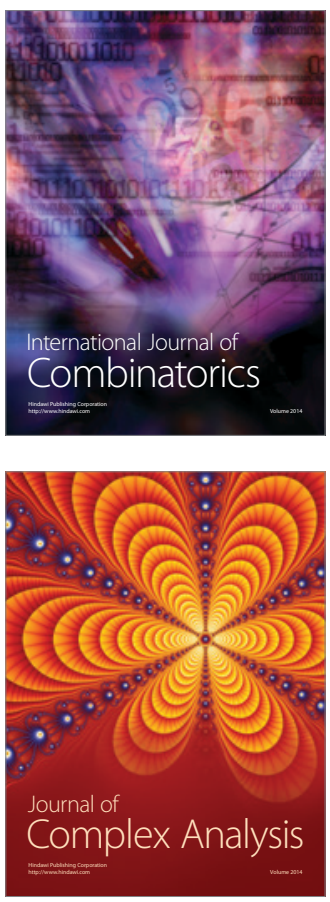

International Journal of

Mathematics and

Mathematical

Sciences
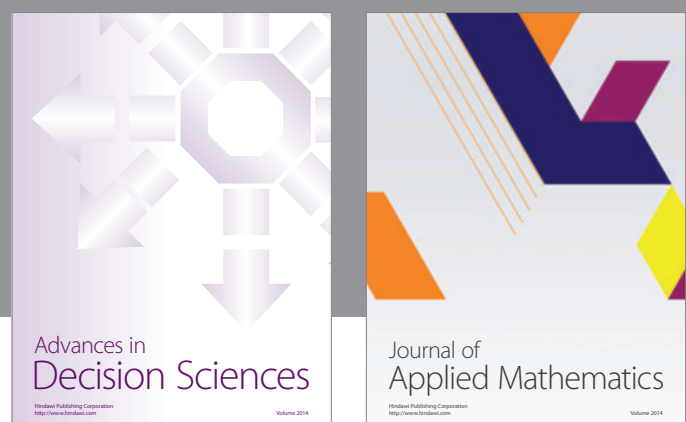

Journal of

Applied Mathematics
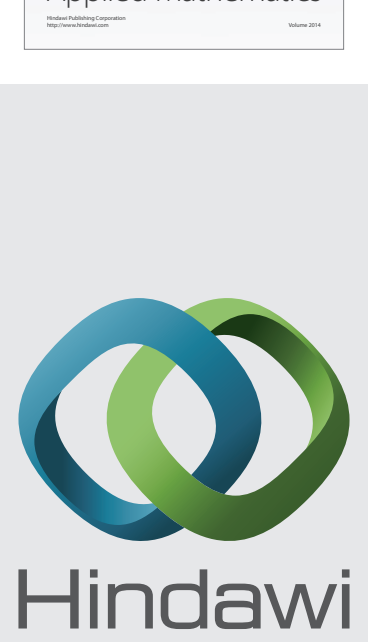

Submit your manuscripts at http://www.hindawi.com
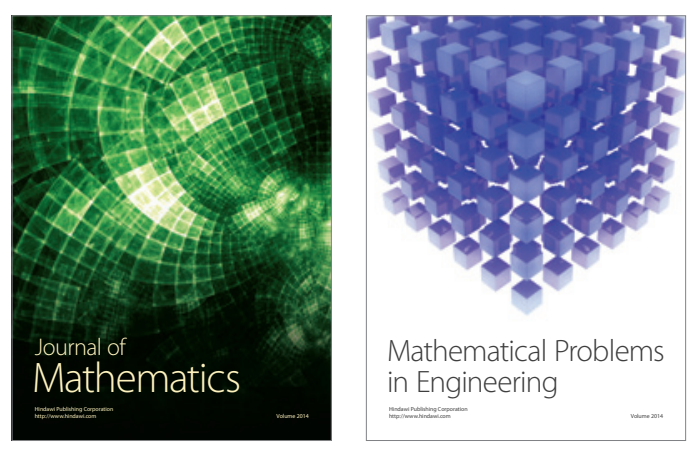

Mathematical Problems in Engineering
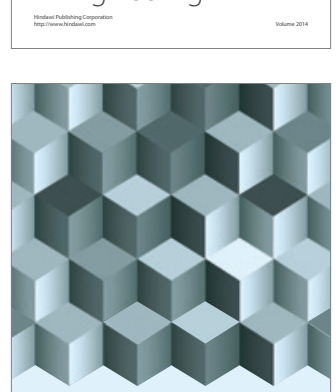

Journal of

Function Spaces
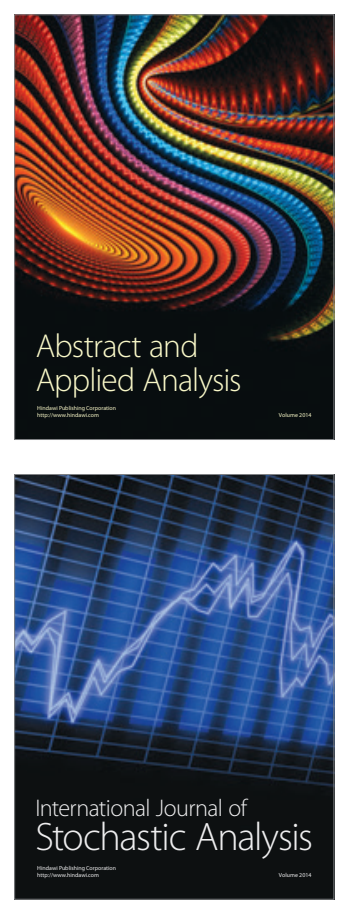

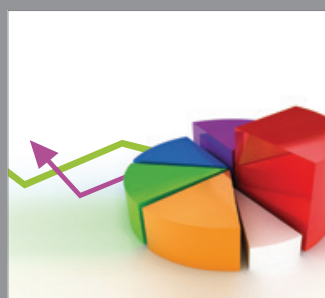

ournal of

Probability and Statistics

Promensencen
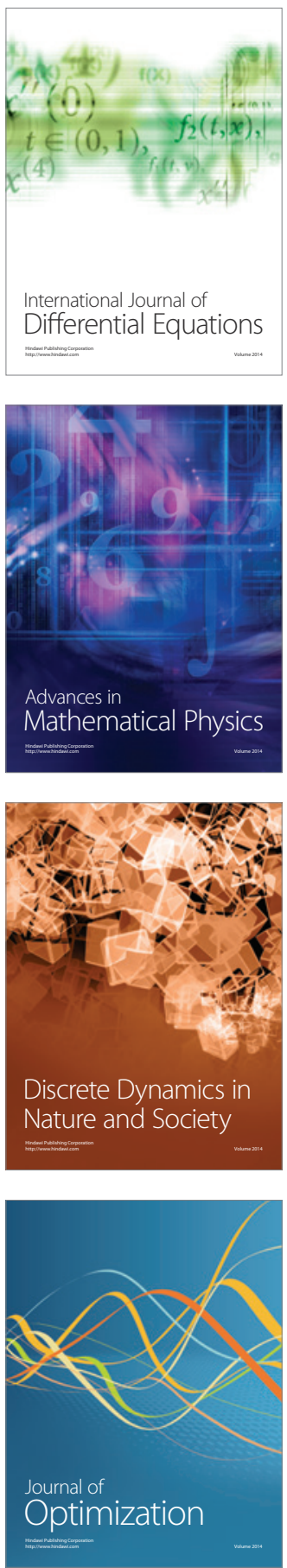